\title{
Numerical Simulation of Small Scale Moored Vessel Motions with Dynamic Mooring Lines
}

\author{
Yuda Apri Hermawan ${ }^{1, a, *}$ and Yoshitaka Furukawa ${ }^{2, b}$ \\ ${ }^{1}$ Department of Maritime Engineering, Graduate School of Engineering, Kyushu University, \\ Fukuoka, Japan \\ ${ }^{2}$ Department of Marine Systems Engineering, Faculty of Engineering, Kyushu University, Fukuoka, \\ Japan \\ a.yuda_apri_h@source.nams.kyushu-u.ac.jp,b.furukawa@nams.kyushu-u.ac.jp \\ *corresponding author
}

Keywords: Small Scale LNG; Small Scale Moored Vessel; Dynamic Mooring Line; Interaction Between Moored Vessel and Mooring Line.

Abstract: Small scale Liquefied Natural Gas (LNG) has been proven as an effective solution to make the natural gas available for the archipelagic area. The small scale LNG is suitable to meet the demand of LNG power plant development at the archipelagic area of Indonesia. Subsequently, small scale moored vessels are needed to support the LNG distributions. To ensure the safety and reliability of the small scale moored vessels, motion analysis of a moored vessel including mooring line motions should be conducted. This motion analysis will become important because of the environmental conditions that tend to be more extreme and deeper water depth comparing with the other parts of Indonesia. It will be a challenge to design the moored vessels operated in the circumstances. Furthermore, accurate estimation of mooring line tension is needed to evaluate the reliability of the mooring lines. The dynamic effects of mooring lines which are often neglected in general mooring analysis methods must be considered. Moreover, the motion analysis solving the moored vessel motion and mooring lines simultaneously should be introduced to consider the interaction between them. In this paper, numerical simulations are conducted to investigate the small scale moored vessel motions in the archipelagic area of Indonesia considering the dynamic effects of mooring lines. Three-dimensional lumped mass method is used to calculate the dynamics of mooring lines while the Manoeuvring Modeling Group model is used to estimate the vessel motions in the horizontal plane. The calculated vessel motions are later on combined with the mooring line motions to introduce the coupledmotion effects between vessel and lines. Finally, the environmental data representing the considered area is adopted to characterize the external forces acting on the vessel. The results indicate that the moored vessel motions reflect the effects of dynamic mooring lines associated with the environmental conditions of the considered area. 


\section{Introduction}

The rapid increase in energy demand is expected over the world in the future as a consequence of population growth. At the same time, the concern of the environmental effects due to the energy consumption becomes more attention. It is generally known that natural gas, the cleanest burning fossil fuel, has propitious environmental property due to its lower $\mathrm{CO} 2$ emission gas comparing with the existing energy resources such as fuel and coal. Then, it is expected to become suitable and effective solution to meet the growing energy demand associated with the constraints limited by environmental issues. According to BP Energy Outlook 2030, similar tendency is reported by Lee et al. [1]. They concluded that the gas demand and production would substantially increase in the future. Therefore, the natural gas will play a major and important role in energy supply chain.

The massive gas demand in the future tend to force the exploitation and supply of gas even at stranded area. The stranded area especially for gas exploitation field is tends to be located at deepwater and far from onshore, then it is not possible to access onshore infrastructures. The installation of offshore pipelines does not seem economical enough for such stranded condition. Meanwhile, aside from the limited access of onshore infrastructure, more attention to acquire gas supply among islands is necessary at remote and archipelagic area. There is essential demand of natural gas such as fuel for LNG power plant. However, these areas are far away from the capital region, lack in infrastructure, spread to several islands and in severe environment conditions. Hence, an appropriate LNG distribution chain should be built to meet the gas demand in the remote and archipelagic area.

Small scale LNG distribution has been proven as an effective solution to make the natural gas available for remote and archipelagic area. As instance, Arthana et al. [2] designed LNG distribution for the eastern part of Indonesia which is one of the remote and archipelagic area in Indonesia and confirmed that the optimum design of LNG distribution for this region can be achieved based on small scale LNG concept. They also concluded that the archipelagic area of Indonesia at where the development of LNG power plant is currently under way is suitable for the small scale LNG supply chain to meet the demand. Therefore, in relation with small scale LNG supply chain itself, inherent supporting facilities such as small scale moored vessel are needed to support the LNG distribution in addition to small scale LNG carrier.

In order to construct offshore facility functioning as a hub for distributing LNG to small and scattered islands, a moored vessel such as small scale Floating Storage Unit (FSU) is generally necessary. On the other hand, mini Floating Storage and Regasification Unit (FSRU) and Floating Power Plant (FPP) are also moored at the final destination area. As the offshore structures which are limited by regulated operation constrains, the safety and reliability of small scale moored vessels become more important. To ensure it, the motion analysis of a moored vessel including mooring line motions should be conducted at design stage. This motion analysis should pay great attention to both environmental conditions that tend to be more extreme and deeper water depth comparing with the other parts of Indonesia. It will be a challenge to design the small scale moored vessels operated in such circumstances.

As for a structure moored by mooring lines, the relation between the motions of a moored vessel and its mooring lines is very tight and interdependent each others. Accurate estimation of mooring line tension is needed in order to ensure the reliability of mooring lines. In order to predict the mooring line tension precisely, the dynamic effects of mooring lines which are often neglected in general mooring analysis methods must be considered. Furthermore, the simultaneous analysis of moored vessel motion and mooring lines which is frequently obtained through uncoupled motion model that solves the motions of floating body and mooring lines separately should be introduced to 
consider the interaction between them. Since the moored vessel motions are influenced by the mooring lines motions and vice versa, this complex condition requires the simultaneous analysis of both structures.

In this paper, the authors conducted numerical simulations to investigate small scale moored vessel motions in the archipelagic area of Indonesia considering the dynamic effects of mooring lines. Three-dimensional lumped mass method is used to calculate the dynamics of mooring lines including the effects of hydrodynamic forces and their elasticity. The calculated mooring line motions are combined with floating body motions in the horizontal plane calculated by mathematical models based on the Manoeuvring Modeling Group (MMG) model to introduce the coupled-motion effects between the moored vessel and mooring lines simultaneously. The environmental conditions including wind and current representing those in considered area a adopted to characterize the external forces acting on the vessel. Simulated results indicate that small scale moored vessel motion in the horizontal plane reflecting the effects of dynamic line tension associated with external forces at the considered area can be predicted by using the developed numerical simulation method.

\section{Equations of Motions}

\subsection{Motion Equation of Moored Vessel}

The motion equations based on Manoeuvring Modeling Group (MMG) model for hydrodynamic forces acting on a ship hull are adopted to introduce the motions of moored vessel in horizontal plane. These equations refer the coordinate systems shown in Fig. 1 . Let $o-x_{0} y_{0}$ be the earthfixed coordinate system and $G-x y$ be the body-fixed coordinate system with the origin at the center of gravity $G$ of the floating structure. Let forces acting on the body in the $x$ and $y$ directions be $X$ and $Y$ and moment around the vertical axis which passes through the center of gravity $G$ be $N$. Wind speed and wind direction are denoted as $V_{w}$ and $v$ while $V_{c}$ and $\alpha$ are current velocity and current direction. $x_{b_{j}}$ and $y_{b_{j}}$ represent the position of attached point of $j$-th mooring line in bodyfixed coordinate system and $\xi_{j}$ is an angle between the mooring line and $x_{0}$-axis. $U$ is velocity of moored vessel and its components in $x$ - and $y$-directions are denoted by $u$ and $v$. Drift angle, heading angle and yaw rate are denoted by $\beta, \psi$ and $r$ respectively with the positive directions shown in Fig. 1. Initial condition was assumed that a ship-shaped floating structure does not have forward speed and stay at a fixed position on the surface of ideal incompressible fluid with external disturbances such as current and wind. The forces acting on the floating structure consist of hull forces $\left(X_{H}, Y_{H}, N_{H}\right)$, wind forces $\left(X_{W}, Y_{W}, N_{W}\right)$ and tension forces due to mooring lines $\left(X_{T}, Y_{T}, N_{T}\right)$. Current forces are included in the hull forces. The equations of motion of a floating structure are expressed as follows:

$$
\left.\begin{array}{r}
\left(m+m_{x}\right) \dot{u}-\left(m+m_{y}\right) v r-\left(m_{x}-m_{y}\right) V_{c} r \sin (\psi-\alpha) \\
=X_{H}+X_{W}+X_{T}, \\
\left(m+m_{y}\right) \dot{v}+\left(m+m_{x}\right) u r-\left(m_{y}-m_{x}\right) V_{c} r \cos (\psi-\alpha) \\
=Y_{H}+Y_{W}+Y_{T} \\
\left(I_{z z}+i_{z z}\right) \dot{r}=N_{H}+N_{W}+N_{T}
\end{array}\right\}
$$

where,

m : mass,

$m_{x}, m_{y}:$ added mass component in $x$ - and $y$-axis directions,

$I_{z Z} \quad$ : moment of inertia,

$i_{z z} \quad$ : added moment inertia

$u, v \quad:$ velocity components in $x$ - and $y$-axis directions,

$\psi \quad$ : heading angle,

$r \quad$ : yaw rate. 


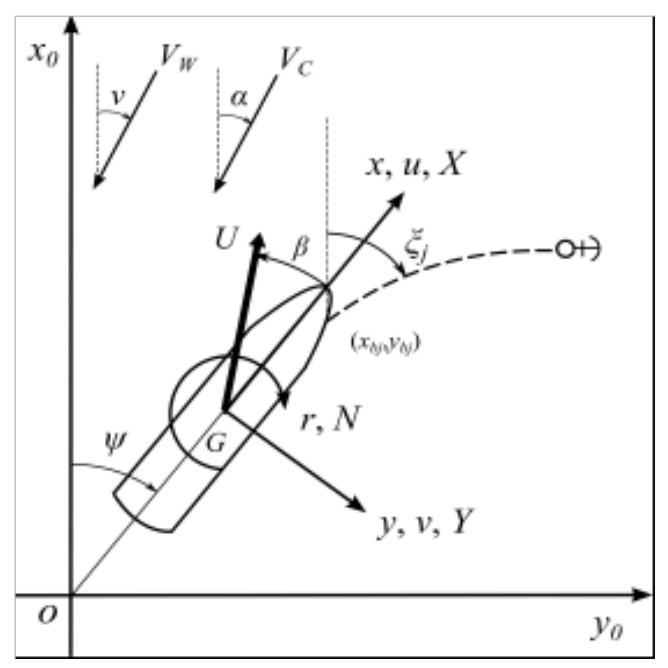

Figure 1: Coordinate systems.

\subsection{Mathematical Model for External Forces}

Hydrodynamic forces acting the hull are expressed as follows according to Yasukawa and Yoshimura [3],

$$
\left.\begin{array}{rl}
X_{H}, Y_{H} & =\frac{1}{2} \rho L d U^{* 2} \times X_{H}^{\prime}, Y_{H}^{\prime}, \\
N_{H} & =\frac{1}{2} \rho L^{2} d U^{* 2} U^{* 2} \times N_{H}^{\prime},
\end{array}\right\}
$$

in which $\rho$ is the water density and $L$ and $d$ are length and draught of a moored vessel respectively. $U^{*}$ represents relative speed over current, $X_{H}^{\prime}$ and $Y_{H}^{\prime}$ are non-dimensional hydrodynamic forces in $x$ - and $y$-directions and $N_{H}^{\prime}$ is non-dimensional yaw moment.

$X_{H}^{\prime}$ is calculated based on mathematical model proposed by Kijima et al. [4],

$$
X_{H}^{\prime}=X_{u u}^{\prime} \cos \beta^{*}\left|\cos \beta^{*}\right|+X_{\beta r}^{\prime} r^{\prime} \sin \beta^{*}
$$

The calculation of $Y_{H}^{\prime}$ and $N_{H}^{\prime}$ is classified into two conditions according to the magnitude of drift angle $\beta$, i.e. for the condition with small drift angle $\left(\beta \leq 30^{\circ}\right)$ and the condition with large drift angle $\left(\beta>30^{\circ}\right)$. It should be noted that drift angle $\beta$ is replaced with relative drift angle $\beta^{*}$ when the influences of current is considered.

$Y_{H}^{\prime}$ and $N_{H}^{\prime}$ for the condition with small relative drift angle $\left(\beta^{*} \leq 30^{\circ}\right)$ are calculated by the following mathematical models presented by Kijima et al. [4]:

$$
\left.\begin{array}{rl}
Y_{H}^{\prime}= & Y_{\beta}^{\prime} \beta^{*}+Y_{r}^{\prime} r^{\prime}+Y_{\beta \beta}^{\prime} \beta^{*}\left|\beta^{*}\right|+Y_{r r}^{\prime} r^{\prime}\left|r^{\prime}\right| \\
& +\left(Y_{\beta \beta r}^{\prime} \beta^{*}+Y_{\beta r r}^{\prime} r^{\prime}\right) \beta^{*} r^{\prime}, \\
N_{H}^{\prime}= & N_{\beta}^{\prime} \beta^{*}+N_{r}^{\prime} r^{\prime}+N_{\beta \beta}^{\prime} \beta^{*}\left|\beta^{*}\right|+N_{r r}^{\prime} r^{\prime}\left|r^{\prime}\right| \\
& +\left(N_{\beta \beta r}^{\prime} \beta^{*}+N_{\beta r r}^{\prime} r^{\prime}\right) \beta^{*} r^{\prime} .
\end{array}\right\}
$$

The formulas proposed by Muto et al. [5] are adopted to obtain $Y_{H}^{\prime}$ and $N_{H}^{\prime}$ for the condition with large drift angle $\left(\beta^{*}>30^{\circ}\right)$, 


$$
\left.\begin{array}{c}
Y_{H\left(r^{\prime}, \alpha\right)}^{\prime}=A_{Y \alpha} r^{\prime 2}+B_{Y \alpha} r^{\prime}+C_{Y \alpha}, \\
N_{H\left(r^{\prime}, \alpha\right)}^{\prime}=A_{N \alpha} r^{\prime 2}+B_{N \alpha} r^{\prime}+C_{N \alpha} .
\end{array}\right\}
$$

Wind forces are estimated according to Fujiwara's model [6], while the tension forces by mooring lines are calculated by the following equations,

$$
\left.\begin{array}{rl}
X_{T} & =\sum_{J=1}^{N}\left\{T_{H_{j}} \cos \left(\xi_{j}-\psi\right)\right\}, \\
Y_{T} & =\sum_{J=1}^{N}\left\{T_{H_{j}} \sin \left(\xi_{j}-\psi\right)\right\}, \\
N_{T} & =\sum_{J=1}^{N}\left\{-T_{H_{j}} \cos \left(\xi_{j}-\psi\right) y_{b_{j}}+T_{H_{j}} \sin \left(\xi_{j}-\psi\right) x_{b_{j}}\right\},
\end{array}\right\}
$$

in which $T_{H_{j}}$ is the horizontal tension of the $j$-th mooring line calculated by dynamic mooring lines methods.

\section{Three-Dimensional Analysis of Mooring Lines}

Three dimensional lumped mass method introduced by Nakajima et al. [7] is used to calculate mooring line tension. In this method, a mooring line is divided into a finite number of elements in which the mass and the forces acting on the element concentrate to each mass point. The elastic deformation of the elements, line-seabed interaction and hydrodynamic drag force due to line motion are included in the calculation methods.

The schematic diagram of the three-dimensional lumped mass is shown in Figure 2. A mooring line consists of $N-1$ mass points, approximating that they are connected by a mass-less linear spring between the mass points. It is assumed that the weight of an arbitrary mass point $j$ is $\delta_{j}$ and tension forces acting on the mass point are $T_{j}$ and $T_{j-1}$ as shown in the Figure 2 . The length including the elongation of each segment is denoted as $l_{j}$. In this calculation method, the mass of the first raised mass point indicated as node $I$ and the last node $N$ are considered 1.5 times than those of others.

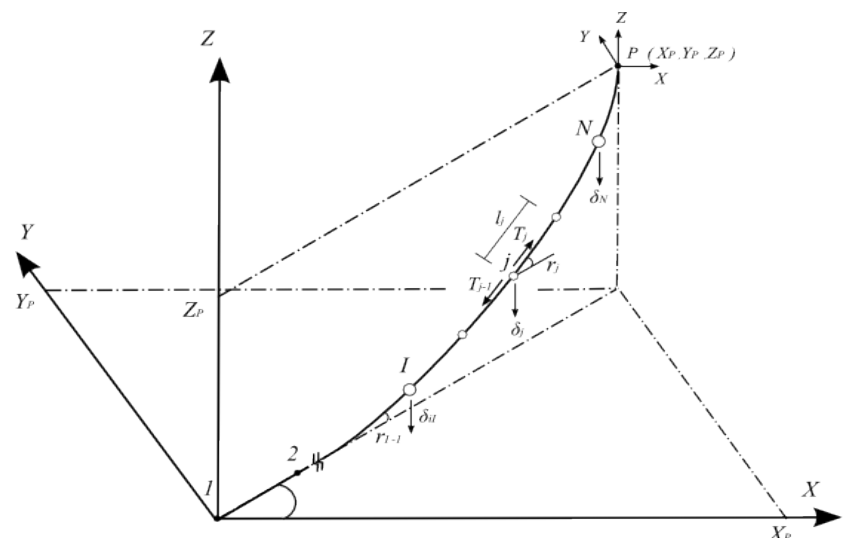

Figure 2: Coordinate systems of mooring line using lumped mass model.

The three-dimensional motion equation of mass point $j$ is given by the following equation, 


$$
\left[\begin{array}{ccc}
I_{1 j} & I_{2 j} & I_{3 j} \\
J_{1 j} & J_{2 j} & J_{3 j} \\
K_{1 j} & K_{2 j} & K_{3 j}
\end{array}\right]\left[\begin{array}{c}
\ddot{x}_{j} \\
\ddot{y}_{j} \\
\ddot{z}_{j}
\end{array}\right]=\left[\begin{array}{c}
F_{x j} \\
F_{y j} \\
F_{z j}
\end{array}\right] .
$$

For the case with the consideration of the elongation, the solution of mooring line motion equation follows the general equation as follows,

$$
\left.\begin{array}{l}
\ddot{x}_{j}^{n}=\left(2 x_{j}^{n+1}-5 x_{j}^{n}+4 x_{j}^{n-1}-x_{j}^{n-2}\right) / \Delta t^{2}, \\
\dot{x}_{j}^{n}=\left(11 x_{j}^{n+1}-18 x_{j}^{n}+9 x_{j}^{n-1}-2 x_{j}^{n-2}\right) / 6 \Delta t^{2},
\end{array}\right\}
$$

where, $n$ represent the time step. From Eq. (8), the displacement of mass point can be obtained as follows,

$$
\left.\begin{array}{rl}
x_{j}^{n+1}= & \frac{5}{2} x_{j}^{n}-2 x_{j}^{n-1}+\frac{1}{2} x_{j}^{n-2} \\
& +\left(R_{j}^{n+1} \cdot T_{j}^{n+1}-P_{j}^{n+1} \cdot T_{j-1}^{n+1}+U_{j}^{n+1}\right) / 2, \\
y_{j}^{n+1}= & \frac{5}{2} y_{j}^{n}-2 y_{j}^{n-1}+\frac{1}{2} y_{j}^{n-2} \\
& +\left(O_{j}^{n+1} \cdot T_{j}^{n+1}-H_{j}^{n+1} \cdot T_{j-1}^{n+1}+V_{j}^{n+1}\right) / 2, \\
z_{j}^{n+1}= & \frac{5}{2} z_{j}^{n}-2 z_{j}^{n-1}+\frac{1}{2} z_{j}^{n-2} \\
& +\left(S_{j}^{n+1} \cdot T_{j}^{n+1}-Q_{j}^{n+1} \cdot T_{j-1}^{n+1}+W_{j}^{n+1}\right) / 2, \\
\quad(j=2 \sim N),
\end{array}\right\}
$$

The tension force $T_{j}$ is given as follows,

$$
T_{j}^{n}=\tilde{T}_{j}^{n+1}+\Delta T_{j}^{n+1} \quad(j=1 \sim N) .
$$

Furthermore, a simultaneous equation with unknown parameters $\Delta T_{j}$ can be derived as follows,

$$
\left[\begin{array}{cccccc}
-\tilde{F}_{2}^{n+1} & \tilde{G}_{2}^{n+1} & 0 & 0 & \cdots & 0 \\
\tilde{E}_{3}^{n+1} & -\widetilde{F}_{3}^{n+1} & \tilde{G}_{3}^{n+1} & 0 & \cdots & 0 \\
0 & \tilde{E}_{4}^{n+1} & -\widetilde{F}_{4}^{n+1} & \widetilde{G}_{4}^{n+1} & \cdots & 0 \\
\vdots & \vdots & \vdots & \ddots & \cdots & \vdots \\
0 & 0 & \cdots & \tilde{E}_{N}^{n+1} & -\tilde{F}_{N}^{n+1} & \tilde{G}_{N}^{n+1} \\
0 & 0 & \cdots & 0 & \tilde{E}_{N+1}^{n+1} & -\widetilde{F}_{N+1}^{n+1}
\end{array}\right]\left[\begin{array}{c}
\Delta T_{1}^{n} \\
\Delta T_{2}^{n} \\
\Delta T_{3}^{n} \\
\Delta T_{4}^{n} \\
\vdots \\
\Delta T_{N}^{n}
\end{array}\right]=\left[\begin{array}{c}
-\widetilde{\Psi}_{2}^{n+1} \\
-\widetilde{\Psi}_{3}^{n+1} \\
-\widetilde{\Psi}_{4}^{n+1} \\
-\widetilde{\Psi}_{5}^{n+1} \\
\vdots \\
-\widetilde{\Psi}_{N+1}^{n+1}
\end{array}\right]
$$

in which respective coefficients are given as follows, 


$$
\left.\begin{array}{rl}
\tilde{E}_{j}^{n+1}= & \frac{\delta \widetilde{\varphi}_{j}^{n+1}}{\delta \tilde{T}_{j-2}^{n+1}}=P_{j-1}^{n+1}\left(\tilde{x}_{j}^{n+1}-\tilde{x}_{j-1}^{n+1}\right) \\
& +H_{j-1}^{n+1}\left(\tilde{y}_{j}^{n+1}-\tilde{y}_{j-1}^{n+1}\right)+Q_{j-1}^{n+1}\left(\tilde{z}_{j}^{n+1}-\tilde{z}_{j-1}^{n+1}\right), \\
F_{j}^{n+1}= & -\frac{\delta \widetilde{\Psi}_{j}^{n+1}}{\delta \widetilde{T}_{j-2}^{n+1}}=\left(P_{j}^{n+1}+R_{j-1}^{n+1}\right)\left(\tilde{x}_{j}^{n+1}-\tilde{x}_{j-1}^{n+1}\right) \\
& +\left(H_{j}^{n+1}+O_{j-1}^{n+1}\right)\left(\tilde{y}_{j}^{n+1}-\tilde{y}_{j-1}^{n+1}\right) \\
& +\left(Q_{j}^{n+1}+S_{j-1}^{n+1}\right)\left(\tilde{z}_{j}^{n+1}-\tilde{z}_{j-1}^{n+1}\right) \\
& +2 \bar{l}^{2}\left(1+T_{j-1}^{n+1} / E \cdot A\right)^{2}, \\
\tilde{G}_{j}^{n+1}= & \frac{\delta \widetilde{\Psi}_{j}^{n+1}}{\delta \tilde{T}_{j-2}^{n+1}}=R_{j}^{n+1}\left(\tilde{x}_{j}^{n+1}-\tilde{x}_{j-1}^{n+1}\right) \\
& +O_{j}^{n+1}\left(\tilde{y}_{j}^{n+1}-\tilde{y}_{j-1}^{n+1}\right)+S_{j}^{n+1}\left(\tilde{z}_{j}^{n+1}-\tilde{z}_{j-1}^{n+1}\right),
\end{array}\right\}
$$

By solving the Eq. (12) repeatedly until $\Delta T_{j}$ converges, the mooring line tension and its motion can be obtained. The tension and position of each mass point is used to calculate next time steps. See the explanation reported by Hermawan et al [8] for the detail of the above calculation method. The attached point of the mooring line is determined by vessel motion equation derived from MMG model.

\section{Result of Small Scale Moored Vessel Motions}

The principle dimensions of a moored vessel and the characteristic of a mooring line are described in Table 1. The principle dimensions of the vessel are assumed based on the common dimensions of small scale moored vessel as described in [9] about $40000 \mathrm{~m} 3$. While the mooring line and anchor characteristics are taken from the common mooring line data used in Indonesian sea.

In present study, a vessel is assumed to be moored with single point mooring system connected on the center line of the vessel. Water depth is set to $500 \mathrm{~m}$ which represents the depth of considered area shown in Figure 3 at where the depth of water is about 400-600 m. The area was selected based on the location of Masela fields, the newest discovered gas field in Indonesia. Wind speed is assumed as $8.78 \mathrm{~m} / \mathrm{s}$ based on database presented in the ftp site of National Oceanic and Atmospheric Administration (NOAA) [10] while the current velocity is assumed as 0.5 knot based on the existing data. The combinations of wind and current direction are adopted according to [11] for non-collinear conditions without consideration of the presence of wave as an early stage. In addition, to consider the influence of wind and current coming from different directions, the combination of current coming from a bow with wind from $30^{\circ}$ is investigated.

Table 1: Principle Dimension and Mooring Line Characteristics.

\begin{tabular}{|c|c|l|c|c|c|}
\hline \multirow{2}{*}{ Principal dimensions } & \multicolumn{3}{|c|}{ Mooring line and anchor characteristics } \\
\cline { 3 - 5 } & & \multicolumn{3}{|c|}{ Mooring line } & \multicolumn{2}{c|}{ Anchor } \\
\hline$L_{O A}$ & $167.600 \mathrm{~m}$ & Length & $1595.00 \mathrm{~m}$ & $w_{a}$ & $17250.00 \mathrm{~kg}$ \\
\hline$L_{P P}$ & $158.805 \mathrm{~m}$ & $w_{c}$ & $210.00 \mathrm{~kg}$ & $\lambda_{a}$ & 7.00 \\
\hline$B$ & $25.898 \mathrm{~m}$ & $D_{c}$ & $102.00 \mathrm{~m}$ & \\
\hline$d$ & $10.773 \mathrm{~m}$ & $E$ & $5.42 \times 10^{9} \mathrm{kgf} / \mathrm{m}^{2}$ & \\
\\
\hline$C_{b}$ & 0.831 & $\lambda_{c}$ & 0.75 & \\
\hline
\end{tabular}




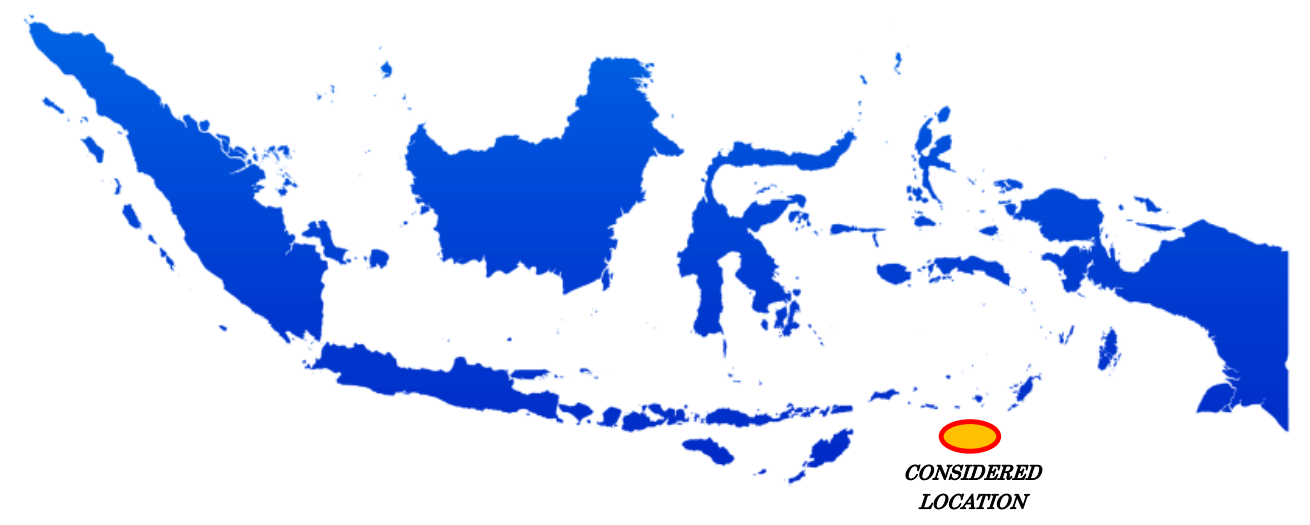

Figure 3: The considered location for conducting analyses

\subsection{Analyses of Small Scale Moored Vessel Motions}

Analyses of a small scale moored vessel motions are observed through the investigation of vessel motion components, i.e. trajectory, heading angle and drift angle which represent the vessel behaviour in given conditions. Simultaneous interactions between the moored vessel and its mooring line are captured by investigating how motion component correlates with each other in relation to how mooring line induces the body motions and vice versa. Calculation results are described as follows.

\section{A. Case 1. Wind and current from same direction}

In this case, current and wind forces are considered as disturbances acting on the moored vessel. Both directions of current and wind are $30^{\circ}$ from bow of the vessel. The results of the Case 1 are shown in Figure 4. The trajectory of the vessel is shown in Figure 4(a). It is observed that the vessel moves backward at the beginning of simulation due to the influence of current and wind forces in $x$ direction, but the motion in longitudinal direction is soon retrained by the presence of mooring line. In this case, the significant force components acting on the vessel is wind force, especially in $x$ direction, as shown in Figure 4(b). The figure presents that the magnitude of tension force of the mooring line is almost equal to that of wind force (abt. $1.4 \mathrm{kN}$ ), but in the opposite direction. Then, the vessel continues to move in lateral direction with gradually changing the heading angle following the effects of current and wind forces.

During the experienced motions, heading angle changes gradually and finally it tends to have the same angle with wind and current directions, $30^{\circ}$. Mooring line angle also changes following the variation of heading angle. It means that the vessel will stop at an equilibrium position at where the heading angle tends to be the same angle with wind after the enough times. This situation can be confirmed by the heading angle presented in Figure 4(c). The final heading angle is $29.747^{\circ}$. It is very close with the angle of wind and current direction, $30^{\circ}$. Meanwhile, the direction of the vessel motion can be investigated by drift angle shown in Figure 4(d). The figure shows that the vessel tends to move with drift angle abt. $120^{\circ}$. It corresponds to the vessel trajectory shown in Figure 4(a).

The three-dimensional motion of mooring line calculated by lumped mass method is shown in Figure 5. From the figure, it is noted that the mooring line motions is reproduced reasonably. In this case, the shape of mooring line changes following the vessel's motions. Especially for the motion of the laying part of the mooring line, the shape of the part changes gradually due to the effects of friction forces between line element and seabed. 


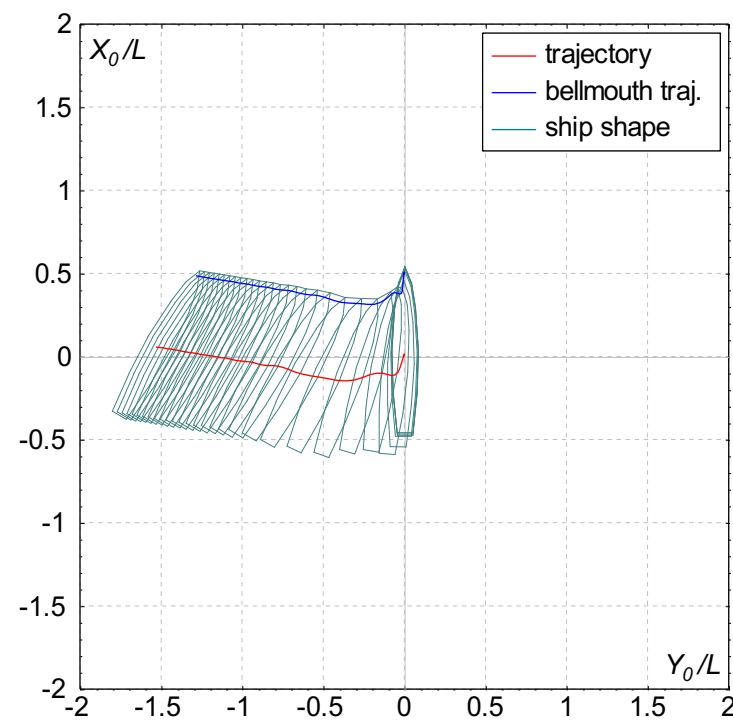

(a) Trajectory

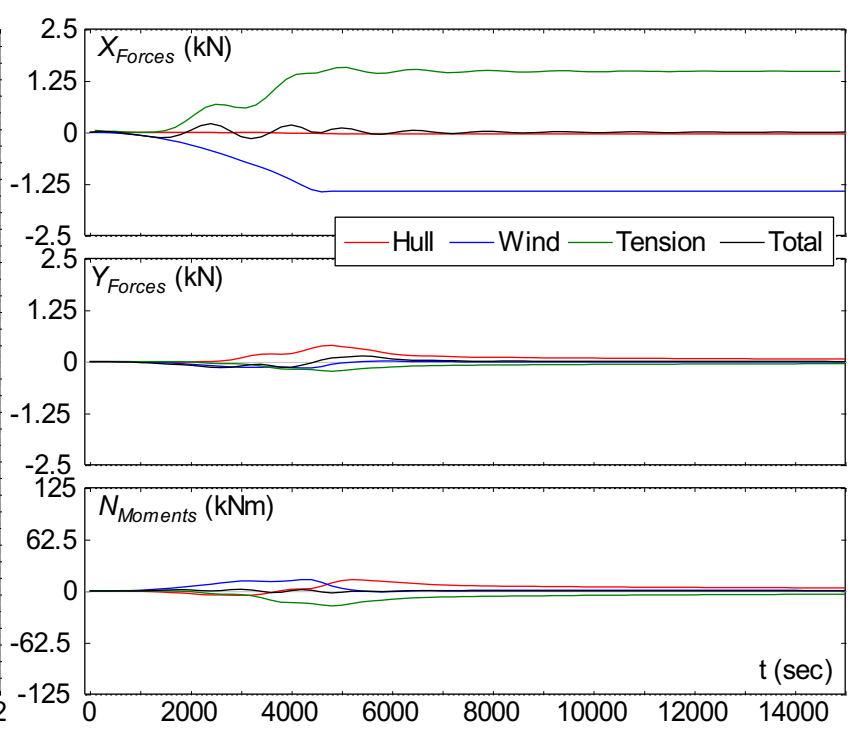

(b) Force components

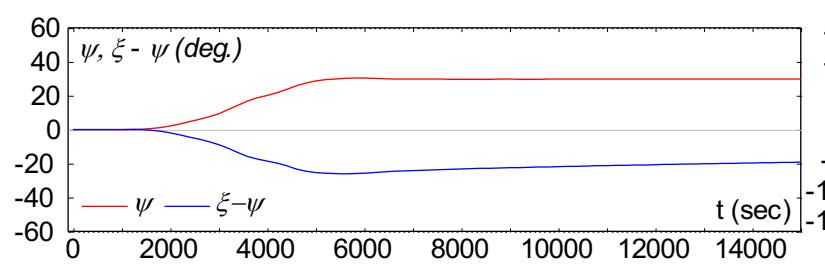

(c) Heading and line angles

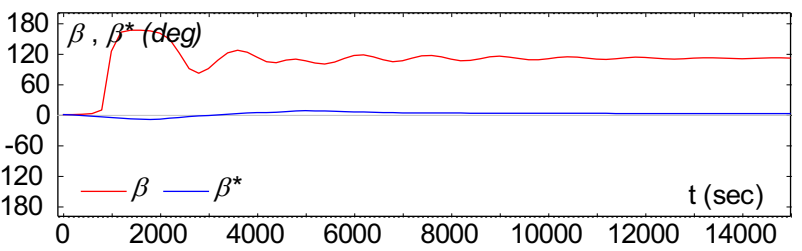

(d) Drift and relative drift angles

Figure 4: Vessel motion results of Case 1.

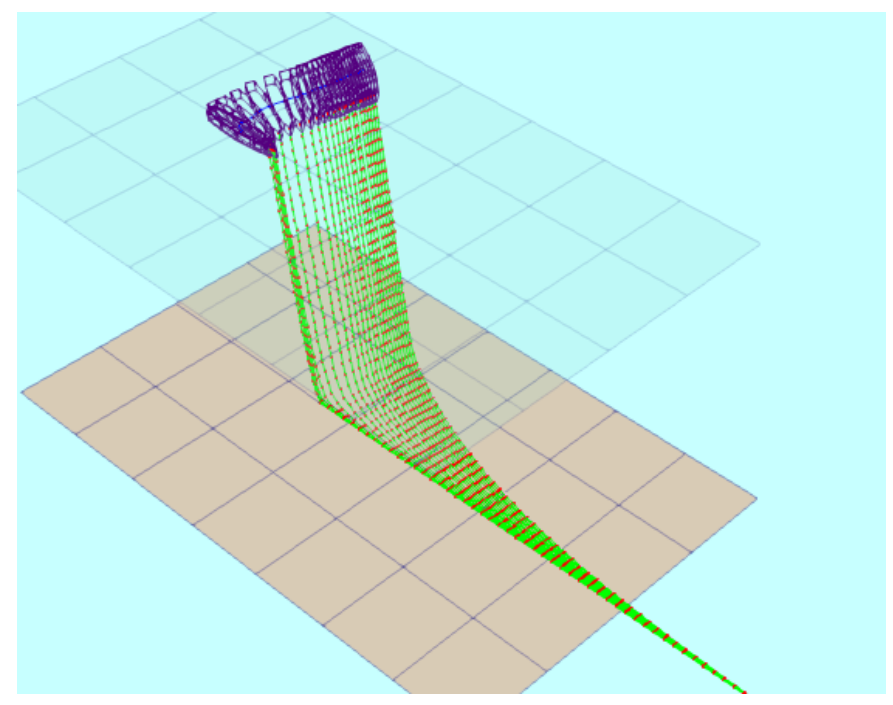

Figure 5: Vessel motions with mooring line motions for Case 1.

According to the results, it can be noted that the coupled dynamic motions of moored structure can be expressed reasonably. At the beginning of simulation, vessel moves under the influences of wind and current which come from same directions. She changes her heading angle following the direction of disturbance forces generated by wind and current. As the position at where the mooring 
line attaches the vessel changes due to the changes of vessel's heading and trajectory, the mooring line tension also simultaneously changes following the change of attached position at the vessel.

\section{B. Case 2. Wind and current from different directions}

To investigate the influence of wind and current coming from different directions, a simulation with wind direction $v=30^{\circ}$ and current direction $\alpha=90^{\circ}$ is conducted according to [11]. Figure 6 shows the moored vessel motion results in the Case 2. In this case, it can be observed in Fig. 6(a) that the vessel tends to move in lateral direction constantly due to the significant lateral force and yaw moment generated by wind and current. It is also confirmed by Figure 6(d) which shows that the vessel tends to move with constant drift angle.

As shown in Figure 6(b), the lateral force and yaw moment are larger than those of the Case 1 (2.02 kN and $0.6 \mathrm{kN}$ for $Y_{H}$ and $Y_{W} ;-61.9 \mathrm{kNm}$ and $-49.9 \mathrm{kNm}$ for $N_{H}$ and $N_{W}$ for the Case 2 while these are almost zero for the Case 1). As the results, the vessel moves with the significant sway and yaw motions. It forces the vessel to move far away from its initial position. The vessel reaches almost $-4 \mathrm{~L}$ in lateral direction with heading angle $49.5^{\circ}$ at the final position as shown in Figure 6(a). It is confirmed by Figure 6(c).

In this case, even though the trajectory of vessel seems far away from the initial position, it can be noted that the vessel will be stop at an equilibrium position at where the total component forces are equal to zero. This condition is achieved due to restoring force given by the mooring line. It can be observed in Figure 6(b) in which the total forces are equal to zero. It means that the tension forces counterbalance the wind and current forces to force the vessel stops at her equilibrium position. In this condition, the vessel has the certain heading angle depending on the direction of resultant forces affected by wind and current conditions. This tendency can be captured by the heading angle shown in vessel trajectory and Figure 6(c). It is also confirmed that the mooring line angle changes following the variation of heading angle.

The motions of moored structure including its mooring line in the Case 2 are illustrated in Figure 7. According to the figure, the shapes of the mooring line change depending on the vessel trajectory as well as the vessel behavior which is imposed by mooring line tension. The simultaneous calculation results indicate the corresponding propensity of moored vessel motion.

\section{Case 3. Wind and current from different direction}

To ensure the capability of the developed simulation code, a simulation with different condition of external disturbances is conducted considering the current coming from bow directions combined with wind from $30^{\circ}$. The results of the Case 3 are illustrated in Fig. 8. In this case, the mooring line tensions can significantly restrain the vessel motions under the combined condition of current and wind. It is noted by Figure 8(a) which shows that the vessel does not move far away from its equilibrium positions (the maximum offsets are $-0.18 \mathrm{~L}, 0.20 \mathrm{~L}$ ). In this case, forces and moments generated by wind and current can be balanced quickly with mooring line tension even at the beginning of the simulation as confirmed in Figure 8(b). Furthermore, at the final stage, similar with other cases, the vessel will stop at the equilibrium position with constant heading angle, i.e. $20.9^{\circ}$ as depicted in Figure 8(c). In relation to the direction of vessel motion, Figure 8(d) corresponds with the vessel shapes conceived in Figure 8(a) in which the direction of vessel motion is changed from port side to starboard side. It is confirmed by Figure $8(\mathrm{c})$ where the drift angle is change from positive to negative sign at $t=3200 \mathrm{sec}$. Moreover, the motions of moored structure including its mooring line in the Case 3 are illustrated in Figure 9. Based on the results, it is easy to confirm that the motion of moored vessel under the influence of wind and current coming from the different directions can be examined by the developed simulation code. The simultaneous interaction 
between body and mooring line motions can addresses which external force has the most dominant influence on the vessel's motion. Three-dimensional mooring line motions induced by vessel motions and vice versa generate the simultaneous motions of moored vessel including its mooring lines.

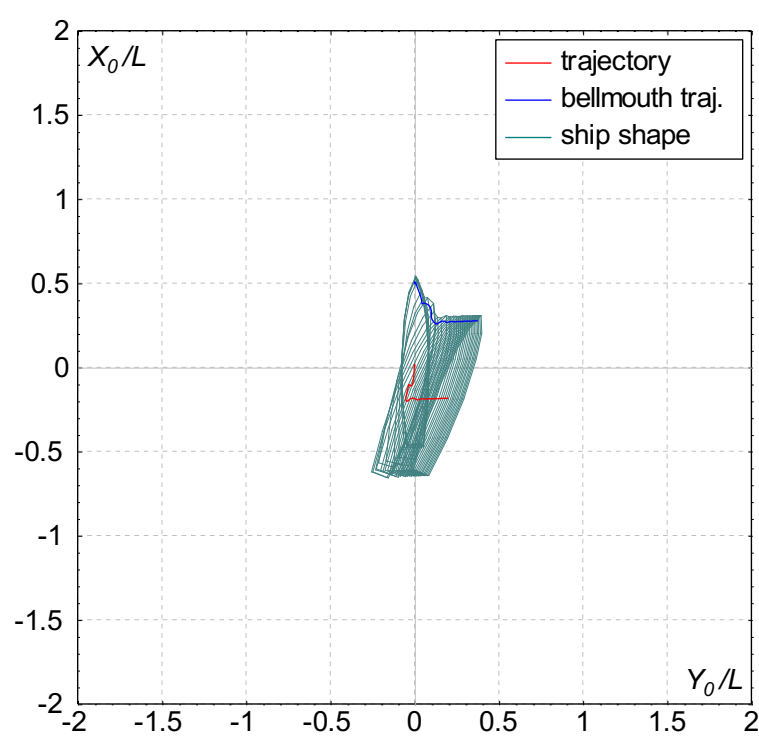

(a) Trajectory

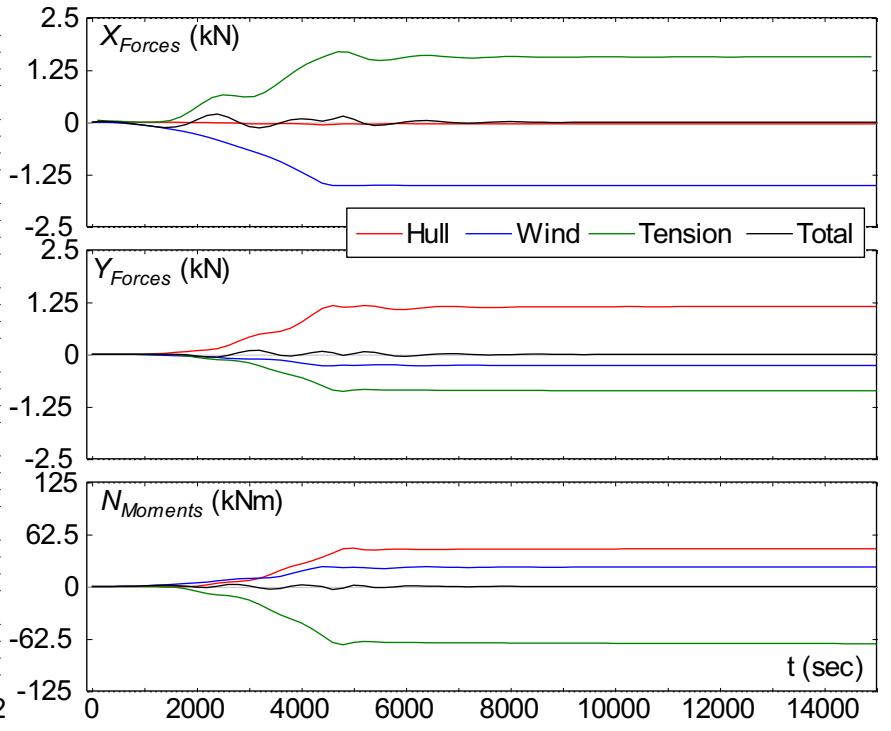

(b) Force components

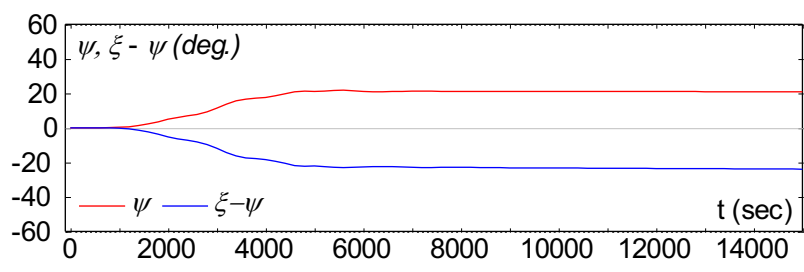

(c) Heading and line angles

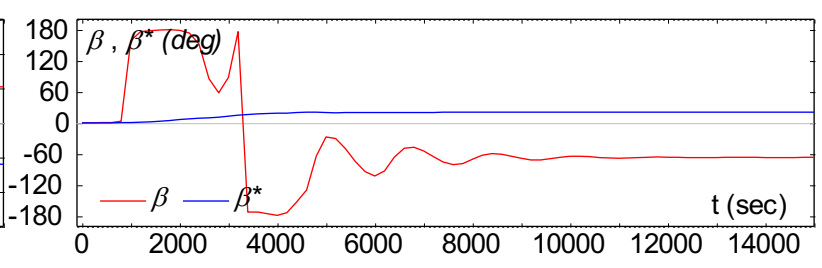

(d) Drift and relative drift angles

Figure 8: Vessel motion results of Case 3.

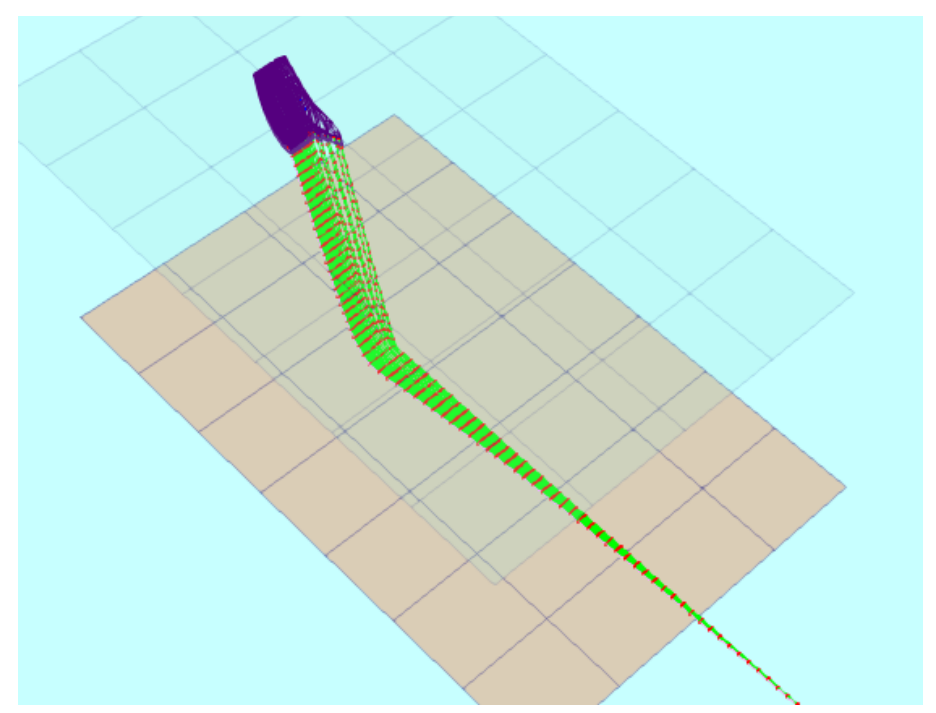


Figure 9: Vessel motions with mooring line motions for Case 3.

\section{Evaluation of Results Analyses}

The special consideration related to small scale moored vessel with single point mooring at the considered area can be investigated by the magnitude of the maximum offset of vessel. It seems to be important relating to the operability of a moored vessel which is limited by the conditions of surrounding area and other structures connected to the vessel.

According to the results of analyses, the vessel offsets tend to be far away from the initial position for the cases required by regulation (Case 1 and Case 2). The far offset of the vessel occurs because the considered area has more extreme environmental conditions than those of others while the vessel dimension is relatively small comparing with the common vessel. It means that the vessel cannot significantly resist the large amount of external forces by herself. The dimension of vessel which is subjected as a small scaled vessel is not enough to withstand the external forces. These external forces seem too strong for a small scaled moored vessel. It is noted that the more efforts are needed in order to reduce the maximum offset of the small scale moored vessel. Varying the mooring system configurations, mooring line characteristics, etc may allow to reduce the moored vessel offsets. These kinds of efforts can be considered for the future works to better ensure the safety operation of small scaled moored vessel.

\section{CONCLUSIONS}

The small scale moored vessel motion in the archipelagic area of Indonesia had been investigated by a numerical simulation which consists of MMG and three-dimensional lumped mass model. Analyses are conducted by considering wind and current which represent the considered location. The combination of both forces directions are introduced based on the classification society, however, neglecting the presence of wave. Another combination also investigated to ensure the capability of simulation code. The results confirm that the motions of small scale moored vessel can be predicted properly including the effect of wind and current forces at such locations and it can be applicable including the dynamic effects of mooring lines. The simultaneous interaction between mooring line and moored vessel can be introduced by this simulation code, even though the wave did not consider yet. To complete this simulation code, the effects of wave forces will be included into simulation code in further work.

\section{References}

[1]. D.H. Lee, M.K. Ha, S.Y. Kim, and S.C. Shin, "Research of Dsign Challenges and New Technologies for Floating LNG, ”Int. J. Nav. Archit. Ocean Eng, Vol. 6. pp. 307-322, 2014.

[2]. K.B. Arthana, K. Sambodho, P.S. Asmara, D. Kristanto, D.S. Antara, "Design of Maritime LNG Distributin for Power Plants in Eastern Part of Indonesia, "Proceeding of the International Symposium on Marine Engineeering (ISME), October 15-19, 2017.

[3]. H. Yasukawa, Y. Yoshimura, Y. "Introduction of MMG Standard Method for Ship Maneuvering Predictions," Journal of Marine Science and Technology, 20:37-52., 2015

[4]. K. Kijima, Y. Nakiri, T. Katsuno, Y. Furukawa. "On the Manoeuvring Performance of a Ship with the Parameter of Loading Condition," Journal of SNAJ, Vol. 168., 1990

[5]. H. Muto, Y. Furukawa, H. Ibaragi, Y. Koyanagi, S. Aramaki, "Mathematical Model for Hydrodynamic Force Acting on a Ship Hull with Large Drift Angle," AMEC., 2010.

[6]. T. Fujiwara, M. Ueno, T. Nimura. "Estimation of Wind Force on the Hull," Proceedings of the Japan Society of Shipbuilding, Vol. 183. 1998.

[7]. T. Nakajima, S. Motora, M. Fujino. "A three-Dimensional Lumped Mass Method for the Dynamic Analysis of Mooring Lines," Proceedings of the Japan Society of Shipbuilding, Vol. 154. 1983. 
[8]. Y.A. Hermawan, Y. Furukawa. "Coupled-Dynamic Analysis of Ship-shaped Floating Structure with Dynamic Mooring Lines in Horizontal Plane," submitted to ISOPE 2018 (not published yet)

[9]. A. Hambucker."Developing an Integrated Small \& Mid-Sized Floating LNG Solution,"2013.

[10]. National Oceanic and Atmospheric Administration wave data:

ftp://polar.ncep.noaa.gov/pub/history/waves/

[11]. Biro Klasifikasi Indonesia. "Guidelines for Floating Production Installations 2013," Vol. III. 